\title{
Entanglement stirred up
}

\author{
Stirring a two-dimensional quantum fluid at just the right frequency causes the particles to develop strong \\ quantum correlations. This could reveal much about the nature of phase transitions.
}

\author{
Jacob A. Dunningham
}

$P$ hase transitions have a profound role in physics. Some are familiar, like an ice cube melting in a drink on a warm day. Others are less familiar, but are responsible for fundamental things such as the masses of particles or the clumpy structure of the universe ${ }^{1}$. Phase transitions have long been studied in fields like cosmology and condensedmatter physics, but more recently atomic physics experiments with Bose-Einstein condensates (BECs) have started to provide a fresh perspective on the topic ${ }^{2}$. In a theoretical study, on page 431 of this issue, Daniel Dagnino and colleagues ${ }^{3}$ show that near the critical point of a phase transition in a BEC, strong quantum correlations develop between the particles making up the ultracold atomic vapour. This offers valuable new insights into the physics underlying a class of phenomena known as quantum phase transitions.

Phase transitions can be defined in different ways, but generally involve abrupt changes in the large-scale properties of a system that are often accompanied by symmetry breaking. They fall into two classes: classical transitions, which are driven by thermal noise, and quantum phase transitions, which take place at zero temperature and can only be accessed by varying an external parameter ${ }^{4}$. BECs are an ideal hunting ground for quantum phase transitions because they have extremely low temperatures and their parameters can be changed with relative ease.

Dagnino et al. ${ }^{3}$ consider a BEC in a twodimensional 'pancake-shaped' trap, which is slowly stirred. The geometry of the stirring potential introduces a gap between the energies of the ground and excited states. This is important because it means that the rate of stirring can be varied while always keeping the system in the lowest energy state. Think of a glass of water filled to the brim: if we move it slowly enough, the surface of the water remains flat, but any sudden movements will cause the water to spill.

In contrast to a classical fluid, a BEC cannot undergo rigid-body rotation when stirred, but can only gain angular momentum by forming quantized vortices beyond some critical velocity. These vortices, which are signatures of superfluidity ${ }^{5}$, are analogous to smoke rings or the behaviour of water when it flows down a sink hole. However, unlike these classical examples, they can only circulate at certain discrete rates. When a superfluid is rotated very rapidly, the number of vortices that appear is comparable to the total number of particles. This case has been studied in some detail because the physics involved is closely related to the quantum Hall effect ${ }^{6}$.

Dagnino et al. ${ }^{3}$ investigate a very different regime, that of slow rotations near the threshold at which the first vortex is formed. Below this threshold, the ground state of the BEC does not rotate, and above it the ground state is a single vortex involving all the particles. Because the system always remains in the ground state, it must undergo a sudden macroscopic symmetry-breaking change as the stirring rate is increased. An interesting question concerns the physics near this critical point. Dagnino et al. address that question by considering the relationship between the full quantum ground state and its meanfield approximation.

Mean-field theory is a technique that has been successfully applied in many areas of physics. The idea is that each particle in a many-body system is treated separately and experiences a mean effect due to all the other particles. This simplifies calculations enormously. Dagnino et al. ${ }^{3}$ show that whereas the mean-field approximation describes BECs well far from the critical point, it breaks down near it. This implies that the particles cannot be treated separately and that there are quantum correlations or entanglements between them.

The association between entanglement and phase transitions ${ }^{7,8}$ has been studied in different systems. However, the precise nature of this relationship is not yet fully understood. For example, what role does entanglement play in a quantum phase transition? And does entanglement provide an efficient way to identify possible phase transitions? Recent work has shown that it is possible to have entanglement without any classical correlations ${ }^{9}$ (between at least three parts of the system). Intriguingly, this means that there could be phase transitions that are revealed only in the quantum correlations but not the classical ones. Studying entanglement could therefore provide a way of detecting phase transitions that is more general than conventional methods.

The scheme of Dagnino et al. ${ }^{3}$ could help to find answers to these questions and an important future development would be to see whether it could be investigated experimentally. One potential obstacle is that the energy gap that protects the ground state from the other levels decreases exponentially with the number of particles. This means that it is likely to be restricted to modest numbers of atoms. Despite that limitation, there should still be scope for uncovering interesting details of the microscopic physics near the critical point. These should lead to general answers to some of the many intriguing questions about phase transitions that are still open.

Jacob A. Dunningham is in the School of Physics and Astronomy, University of Leeds, Woodhouse Lane, Leeds, LS2 9JT, UK.

e-mail: j.a.dunningham@leeds.ac.uk

\footnotetext{
References

1. Coles, P. \& Lucchin, F. Cosmology: the Origin and Evolution of Cosmic Structure (Wiley, 2002).

2. Bloch, I., Dalibard, J. \& Zwerger, W. Rev. Mod. Phys. 80, 885-964 (2008).

3. Dagnino, D., Barberán, N., Lewenstein, M. \& Dalibard, J. Nature Phys. 5, 431-437 (2009).

4. Sachdev, S. Quantum Phase Transitions (Cambridge Univ. Press, 2000).

5. Madison, K. W., Chevy, F., Wohlleben, W. \& Dalibard, J. Phys. Rev. Lett. 84, 806-809 (2000)

6. Cooper, N. R. Adv. Phys. 57, 539-616 (2008).

7. Wu, L.-A., Sarandy, M. S. \& Lidar, D. A. Phys. Rev. Lett. 93, 250404 (2004)

8. Osterloh, A., Amico, L., Falci, G. \& Fazio, R. Nature 416, 608-610 (2002).

9. Kaszlikowski, D., Sen(De), A., Sen, U., Vedral, V. \& Winter, A. Phys. Rev. Lett. 101, 070502 (2008).
} 